\title{
Commentary on Mari Romarheim Haugen: Investigating Periodic Body Motions as a Tacit Reference Structure in Norwegian Telespringar Performance
}

\author{
MURIEL SWIJGHUISEN REIGERSBERG [1] \\ University of Sydney
}

\begin{abstract}
This study investigates periodic body motion (foot-tapping and vertical hip motions) as a reference structure in a specific style of traditional Norwegian dance music called telespringar. The intimate relationship between music and motion is often highlighted in rhythm studies of telespringar, so this study encompasses both sound and motion analyses. It is based on a motion capture study of three telespringar performers, examining the foot-stamping of one fiddler and vertical hip motions of two dancers. The results indicate that rather than being primarily derived from the sound itself, the underlying rhythmic structures in telespringar depend upon a shared and embodied knowledge of the underlying asymmetrical reference structure that is implicit in the production and perception of telespringar. I explore here how the methodology used by the author might be further justified and explored. I will also propose ways in which the article could be made more accessible to researchers who question the universality of research outcomes arrived at using laboratory-based experimental settings.
\end{abstract}

Submitted 2016 October 23; accepted 2016 December 21.

KEYWORDS: telespringar, motion capture, dance, music, asymmetrical meter

\section{KEY POINTS OF THE ARTICLE}

ONE of the main contributions of this paper is an alternative interpretation of the correspondence between the underlying beat level of a fiddler's foot stamping and dancers' libration patterns in the Norwegian folk genre telespringar. The performance of the telespringar genre uses two dancers and a fiddler, the later stamping a rhythmic pattern with their feet whilst performing a complex, highly ornamented fiddle pattern during the dancing. The foot stamping occurs on the first and second beats and occasionally on the third beat. The stamp on the first beat is considered the strongest, and the stamp on the third beat, the weakest (Kvifte, 1999; Blom, 2006). The underlying beat level (meter), the author has found, is asymmetrical and indicated by the fiddler's foot stamping. This finding accords with those of others (Kvifte, 1983; Blom, 2006; Ahlbäck, 2003). The author also points out that the underlying pulse in telespringar should be understood as asymmetrical in and of itself (Groven, 1971; Blom, 1981; Kvifte, 2007; Johansson, 2009; Haugen, 2015) as opposed to being a deviation from an underlying isochronous pulse.

Although the dancing in telespringar is improvised, it can be characterised by three main variants in motion pattern: a winding part; an unfastened part and a couple turning part (Bakka et al., 1995). Blom's libration hypothesis $(1981,1993,2006)$ suggests that telespringar dancers perform two down/up motions in every measure: the first down/up motion corresponding with the duration of the first beat; the second down motion corresponding with the duration of the second beat and the second up motion corresponding to the duration of the third beat. Blom labels this the patterned libration of the body's center of gravity showing how this libration pattern can be represented as straight lines between the turning points (peaks and troughs) in the dancers' libration curve. Libration patterns are thought to be style specific and directly linked to the musical meter (Blom, 1981).

The findings of this author's study, however, do not support this hypothesis. Instead, the author argues, the dancers' libration patterns do not seem to consist of two up/down motions in every measure. The author's laboratory-based motion capture analysis study of the dancers' libration curves, shows that 
it is not the turning points in the libration curve that correspond to the fiddler's foot stamping. Instead it is the libration shape that corresponds to the beat duration patterns indicated by the fiddler's foot stamping. The duration of the first beat corresponds to a vertical S-shape; the duration of the second beat corresponds to the deepest down/up motion; and the duration of the third beat corresponds to the final short up/down motion.

\section{METHOD}

Using an optical infrared motion capture system and synchronizing this with recorded sound and video recordings the author analyzed the relationship between the foot stamping and dance, relating it back to the perception of rhythm. A total of ten recordings with three different sessions were carried out. In the first session (I) the fiddler was instructed to play three different tunes: two tunes deemed suitable for dancing (Urjen and Sud-Kåsen) and one tune not suitable for dancing (Jøronatten). The fiddler was also asked to perform the two dancing tunes in both, what the author calls, a concert version and in a dance version. The dance versions of Urjen and Sud-Kåsen were used as play back in session two (II). In the second session the dancers were recorded alone. In session three (III) the fiddler and dancers were recorded whilst performing together. In this submission, the author addresses the findings of session III. All ten recordings were carried out in a motion capture laboratory. Whilst this is not stated, it is assumed that in all cases the same dancers and fiddler were used to keep the variables stable.

\section{ISSUES FOR CONSIDERATION AND FUTURE ENQUIRY}

My own contribution here will be to suggest a variety of ways in which articles such as these might be made more accessible and informative to a readership which questions the universal applicability of labbased studies to genres and humanity. I argue that this is desirable because it will entice researchers from a variety of backgrounds to collaborate more, or to apply new(er) methods to the analysis of music. I suggest that it is through highlighting those areas where a study is (for very valid reasons) restricted, that new knowledge and collaborations may arise which address this valid limitation [2].

One area of considerable interest for example, would be to further justify and explicate the chosen method, motion capture, showing why it might be preferable to another and how it advances knowledge in the field. Motion capture analysis is now commonly used in psychology, but less so in, for example, ethnomusicology, at least in the UK. Presumably motion capture yields more accurate results than say, transcription. Perhaps this is the case because it eliminates human perception from the analytical process. As Haugen (2016) points out, rhythmic perception of meter depends on the perceived pulse and downbeat. It is also influenced by the familiarity of the listener with the genre in question. Haugen references Clayton's work, who describes intra-individual rhythmical entrainment as occurring within a person. Intra-individual entrainment is responsible for the perception of musical meter. Interindividual musical entrainment is what determines the dynamics of physical coordination between people (Clayton, 2012, 2013). Polak (2015), commenting on Goldberg's study of Aksak meter in southeastern Europe (2015) also casts doubt on the widely-held belief that metric regularity strongly privileges vertical symmetry and iso-periodicity. The recordings analysed by Goldberg present an asymmetric rhythmic mode, non-isochronous beat sequence, and asymmetric metric hierarchy. This rhythmic complexity appears not to influence the fluidity of the performances, suggesting that the perception of meter is not negatively affected despite the absence of regularity, vertical symmetry and iso-periodicity. This in turn may mean, argues Polak, that previous assumptions are culturally biased. Looking at it differently, I would suggest here that these assumptions are influenced by an analyst's own intraindividual rhythmical entrainment history, and therefore are not entirely invalid when exploring human musical perception.

This being the case, it could follow that a researcher familiar with a genre, say through performance, or regular listening, might be more adept at transcribing or analysing it using nontechnological methods, if they know how to transcribe. It may therefore be preferable to use motion capture techniques in some cases, where a researcher is less than familiar with a genre or does not have the skill to transcribe what is a complex genre. Understanding why motion capture is the preferred method, and its pros and cons, in turn, might encourage other (ethno) musicologists to engage with it and may increase levels of interdisciplinary enquiry, using a variety of methods.

Another methodological question arises when we look at other rhythmical movements occurring during the performance of Norwegian folk dance. Blom and Kvifte (1986) in the journal Ethnomusicology, study the Norwegian folk genre, gangar. They use transcription rather than motion 
capture to arrive at their conclusions. Blom and Kvifte debate how musical perception of rhythm, pulse and meter might be either emic (from the within a culture) or etic (from without a culture) and how it may or may not be related to dance movements and personal, regional and genre variances.

In the Blom and Kvifte (1986) article the authors include an analysis of the bowing movements and patterns of the fiddler as well as notating and transcribing the music and foot tapping of the fiddler. They combine these with ethnographic information to arrive at conclusions about meter and perception. The authors argue that the bowing of the fiddler is what gives movement to the empty foot tapping, suggesting that the bowing may have an important part to play in metrical and rhythmic formation and analyses, as well as rhythmic perception. It would be interesting to include the fiddler's bowing phrases and movements as an additional way of analyzing telespringar using motion capture.

Including the fiddle melody and phrasing in the analysis of libration movements is more complex. The bowing and Hardanger fiddle sound in telespringar is highly ornamented. Haugen demonstrates this using visualisations of the telespringar sound, plotting the amplitude waveforms and envelope curves against one another, showing fluctuation in energy and the spectral flux. A complex domain onset detection function (Complex DF) (Duxbury et al., 2003) implemented in Sonic Visualiser was applied to the sound signal which indicated important changes in the energy and phase domain combined. Haugen then uses established methods to try and unpick what is happening rhythmically, but concludes that: "When one listens to the recordings of Sud-Kåsen and Urjen, one perceives a beat level in the music to be synchronized with the fiddler's foot stamping, but any attempt to determine beat positions based on the physical sound signal proved fruitless.” It is not clear here whether transcription might have yielded better results than the waveform analysis; whether perhaps a 'mixed methods' approach using transcription and motion capture analysis could have been employed to address this issue; or whether another researcher might have found it easier to determine beat positions because of their intra-individual rhythmical entrainment history. The researcher here, could be a variable in the analytical process (see also Jairazbhoy, 1977). More methodological detail might be provided in future studies to explicate what other avenues could be worth investigating.

Other comparative studies could also be done to investigate differences in methodological effectiveness of various analytical approaches across various folk genres. For example: Blom and Kvifte (1986), using transcription, investigate the commetric (with the beat) and contrametric (against the beat) facets of gangar. They debate whether, depending on how one analyses the music in relation to movement (foot tapping and/or bowing), a commetric or conmetric pattern can be inferred. That is to say, the tapping/ bowing coincide, or they do not. Given that gangar is a related musical style to telespringar, it may be worth re-investigating the Blom and Kvifte (1986) study using motion capture techniques to see whether the question of rhythmical perception of gangar can be informed by this method. Vice versa, it would be interesting to explore if transcription and the inclusion of bowing movements and an analysis of these alongside foot tapping might yield useful results for telespringar. If so, this would have positive implications for research methodologies and musical analyses of regional styles.

Lastly, where method is concerned, it could be helpful to explain why three separate settings were used to record the music: (a) the fiddler on their own; (b) the dancers alone; (c) the fiddler and dancers together. Haugen also mentions a 'concert version'. It is not entirely clear how a concert version varies from other types of rendition, seeing as all studies discussed in this article took place in a laboratory. Presumably the same three performers were used for all experiments, and all are accomplished telespringar experts of a certain standard. Whilst this approach does ensure that the variables remain controlled, to enhance interdisciplinary engagement, it would be important to acknowledge that the results might not be ecologically valid. Personal ability and performative style outside the laboratory were not explored. Given that telespringar dancing relies heavily on improvisation, it might be interesting to see whether the differences between Haugen and Blom's findings are in any way influenced by performative variability and expressivity of individual dancers and fiddlers. It could be useful to replicate the study with different dance pairs, fiddlers, and in context to see what this yields.

Another interesting area of enquiry, unrelated to method, might include developing some links to performance studies and explorations of expressivity in the telespringar genre. One might examine how the rhythmic complexity and its embodiment are experienced and explained by the dancers and fiddlers and how this influences inter-personal rhythmic performance. Clayton (2015) for example, uses this approach combining studies of performer analyses and qualitative data with quantitative information, in order to investigate how the performers describe their perception of asymmetrical reference structures. 


\section{CONCLUSION}

In all, studies such as Haugen's are fascinating in that they allow us to interrogate, as a body of musicological researchers, how we can work collaboratively towards solving the questions we have about the human capacity to create organized sound. Many of these studies raise interesting queries about the universality of musical ability and perception and whether perhaps we are too quick to extrapolate results or draw conclusions based on smaller sample-sizes, or too hasty at negating the validity of laboratory-based experiments. They require that we not only closely examine our methods, but also factor the researchers themselves in to the experimental equation as being a variable. A researcher or performer's intra-personal entrainment may well impact on the analytical outcome of the experimental results. This, interestingly, does not invalidate the results themselves, in that they do represent ways in which music is perceived by a certain group of people, or even by a single person, namely the researcher. The question then arises however, whether information about musical perception can ever be demonstrably, universally valid. My suggestion here is that if musical (including) rhythmical perception and embodiment are indeed culturally determined and influenced by a person's musical performance history, we have many more experiments to do. These must be replicated across a variety of cultures, by a variety of different researchers to explore what is universal and what is culturally or even personspecific. Therefore, collaboration is essential. One way of encouraging such collaboration is by being clear about the merits of specific methods and explicating their usage in an experimental context. Another is through being forthright about the limitations of studies that are undertaken in laboratory settings or in performative settings, whilst also acknowledging that these same studies have valuable insights to offer which can inform new ideas about music and humanity's perception thereof. It is through this type of clarity and transparency that both qualitative and quantitative research can be used together in studies which explore human musicality.

\section{NOTES}

[1] Correspondence can be addressed to: Dr, Muriel E. Swijghuisen Reigersberg, The University of Sydney. E-mail: muriel.swijghuisenreigersberg@sydney.edu.au.

[2] It is of course possible that the Norwegian language items referenced by the author cover this ground as well. My lack of proficiency in Norwegian prevents me from checking this, so I raise it here.

\section{REFERENCES}

Ahlbäck, S. (2003). About asymmetrical beat in the polska. In M. Ramsten (Ed.), The Polish dance in Scandinavia and Poland (vol. 17, pp. 165-180). Stockholm: Svenskt visarkiv.

Bakka, E., Aksdal, B., \& Flem, E. (1995). Springar and pols: Variation, dialect and age: Pilot project on the methodology for determining traditions structures and historical layering of old Norwegian couple dances. Trondheim: Rådet for folkemusikk og folkedans, the Rff Centre.

Blom, J.P. (1981). The dancing fiddle: On the expression of rhythm in Hardingfele Slătter. In J. P. Blom, S. Nyhus, \& R. Sevåg (Eds.), Norsk Folkemusikk (vol. 7, pp. 305-312). Oslo: Universitetsforlaget.

Blom, J.P. \& Kvifte, T. (1986). On the Problem of Inferential Ambivalence in Musical Meter. Ethnomusicology, 30 (3), 491-517. https://doi.org/10.2307/851591

Blom, J.P. (1993). Rytme og frasering—forholdet til dansen. In B. A. Nyhus \& S. Nyhus (Eds.), Fanitullen. Innføring i norsk og samisk folkemusikk (pp. 161-184). Oslo: Universitetsforlaget.

Blom, J.P. (2006). Making the music dance: Dance connotations in Norwegian fiddling. In I. A. Russell, M.A. (Ed.), Play it like it is: Fiddle and dance studies from around the North Atlantic (vol. 5, pp. 7586). Aberdeen: The Elphinstone Institute, University of Aberdeen. 
Clarke, E. \& Cooke, N. (Eds.) (2004). Empirical Musicology: Aims, Methods, Prospects. New York: Oxford University Press. https://doi.org/10.1093/acprof:oso/9780195167498.001.0001

Clayton, M. (2012). What is entrainment? Definition and application in musical research. Empirical Musicology Review, 7 (1-2), 49-56.

Clayton, M. (2013). Entrainment, ethnography and musical interaction. In M. Clayton, B. Dueck \& L. Leante (Eds.), Experience and meaning in music performance (pp. 17-39). New York: Oxford University Press. https://doi.org/10.1093/acprof:oso/9780199811328.003.0002

Clayton, M. (2015). Aksak Patterns and Entrained Interaction in Transylvanian Village Music. Empirical Musicology Review, 10 (4). 292-301. https://doi.org/10.18061/emr.v10i4.4886

Cooke, N. (2008). We are all (ethno)musicologists now. In H. Stobart (Ed.), New (Ethno) Musicologies (pp. 48-70). Maryland, Toronto, Plymouth: Scarecrow Press.

Duxbury, C., Bello, J. P., Davies, M., \& Sandler, M. (2003). Complex domain onset detection for musical signals. Paper presented at the Proc. Digital Audio Effects Workshop (DAFx).

Goldberg, D. (2015). Timing variations in two Balkan percussion performances. Empirical Musicology Review, 10(4), 305-328. https://doi.org/10.18061/emr.v10i4.4884

Groven, E. (1971). Musikkstudiar-ikkje utgjevne før. 1. Rytmestudiar. In O. Fjalestad (Ed.), Eivind Groven. Heiderskrift til 70-årsdagen 8. oktober 1971 (pp. 93-102). Oslo: Noregs boklag.

Haugen, M. R. (2015). Asymmetrical meter in Scandinavian folk music and dance: A case study of Norwegian telespringar. In A. L. J. Ginsborg, M. Phillips, and S. Bramley (Eds.), Proceedings of the ninth triennial conference of the European Society for the Cognitive Sciences of Music (ESCOM) (pp. 432-436). Manchester, UK: Royal Northern College of Music.

Haugen, M. R. (2016). Investigating Periodic Body Motions as a Tacit Reference Structure in Norwegian Telespringar Performance. Empirical Musicology Review, 11(3-4), 272-294. https://doi.org/10.18061/emr.v11i3-4.5029

Jairazbhoy, N. (1977). The "Objective" and Subjective View in Music Transcription. Ethnomusicology, 21(2), 263-273. https://doi.org/10.2307/850947

Johansson, M. (2009). Rhythm into style: Studying asymmetrical grooves in Norwegian folk music. Ph.D. dissertation, University of Oslo.

Kvifte, T. (1983). Om flertydighet i opplevelse av metrum. Studia musicologica Norvegica (trykt utg.) $9,27-42$.

Kvifte, T. (1999). Fenomenet “asymmetrisk takt” i norsk og svensk folkemusikk. Studia musicologica norvegica, 25, 387-430.

Kvifte, T. (2007). Categories and timing: On the perception of meter. Ethnomusicology, 51(1), 64-84.

Polak, R (2015). Pattern and Variation in the Timing of Aksak Meter: Commentary on Goldberg. Empirical Musicology Review, 10 (4), 329-340. https://doi.org/10.18061/emr.v10i4.4883 
Stock, J. (1997). New Musicologies, Old Musicologies: Ethnomusicology and the Study of Western Music. Current Musicology, 62, 40-68. 